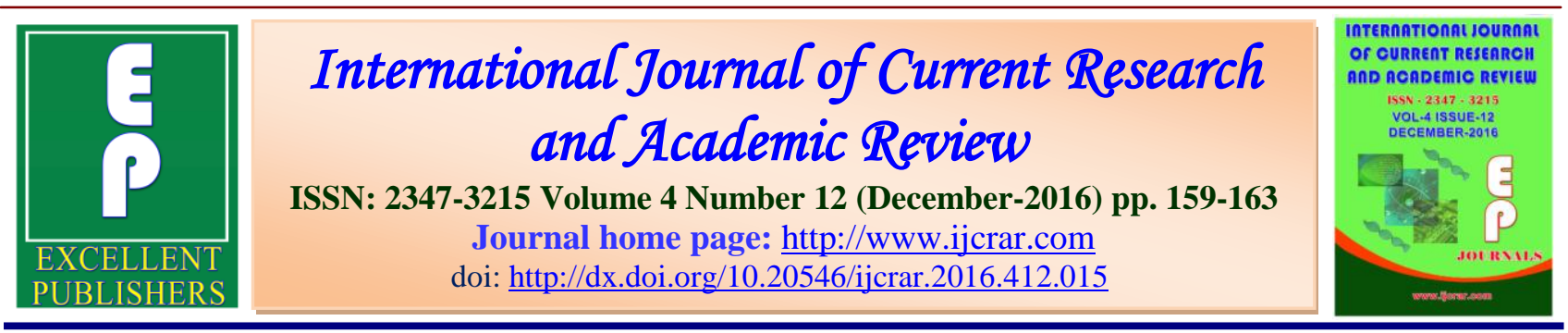

\title{
Agenesis of Permanent Mandibular Incisors: Case Series
}

\section{Savitha Sathyaprasad and Aiswarya Mohan*}

Department of Pedodontics and Preventive Dentistry, KVG Dental College and Hospital, Sullia, India

\section{*Corresponding author}

\begin{tabular}{l|l} 
KEYWORDS & A B S T R A C T
\end{tabular}

Agenesis, Congenitally missing teeth, Mandibular incisors, Etiology, Management.

Congenitally missing teeth are commonly encountered by dental practitioners on routine oral examination. Well documentation of such condition becomes necessary. This paper highlights three case of mandibular incisor agenesis to enrich the knowledge about congenitally missing teeth.

\section{Introduction}

Dental agenesis is the most common developmental anomaly encountered. Missing teeth have been explained under various terms in literature which includes anodontia, hypodontia, oligodontia, aplasia of teeth, congenitally missing teeth, absence of teeth, and agenesis of teeth. Oligodontia and hypodontia are two relative terms in which congenital absence of six or more teeth excluding molars refers to oligodontia and congenital absence of teeth less than six in number excluding molars refers to hypodontia. Maxillary lateral incisors followed by maxillary second premolars and manibular central incisors is the order of occurrence of congenitally missing teeth. There may be unilateral or bilateral absence of teeth. ${ }^{1}$ In primary dentition the prevalence is approximating to $0.1-0.9 \%$ when compared to $2-10 \%$ in permanent dentition. ${ }^{2}$ studies have reported the strong influence of ethinicity on its prevalence ${ }^{3,4}$, as in Caucasian populations the most commonly congenitally missing teeth are either the mandibular second premolars ${ }^{3}$ or the maxillary lateral incisors. ${ }^{5}$ Niswander and Sujaku and Davis showed that the mandibular incisors were the most commonly missing teeth in Japanese as well as Chinese populations. Endo et $a l^{4}$ has reported that the characteristic of hypodontia in a Japanese population compared with foreign populations was a higher prevalence of mandibular lateral incisor agenesis in children with minor hypodontia. Congenital absence of mandibular incisors has exhibited racial ethnicity towards Japanese, Chinese and Korean population. Females have shown higher predilection than males. ${ }^{6}$ 
Int.J.Curr.Res.Aca.Rev.2016; 4(12): 159-163

Teeth agenesis results in malocclusion (which itself can lead to mastication problems $)^{7}$, periodontal damage, lack of alveolar bone growth, reduced chewing ability, inarticulate pronunciation, changes in skeletal relationships and an unfavorable appearance ${ }^{8}$, most of which need rather costly and challenging multidisciplinary treatments. Documentation of such case reports is necessary due to its rarity, to provide a review to minimize the clinicians challenge in diagnosing such cases and thus helpful in providing a multidisciplinary approach in treating the patient.

\section{Case Reports}

\section{Case 1}

An 11-year-old male patient reported to the department of Pedodontics and preventive dentistry with chief complaint of spacing between the lower teeth and wanted to get it orthodontically corrected. On examination patient's height, weight, built and nourishment corresponds with the chronological age. Intra oral examination of the lower arch, revealed the presence space between the permanent mandibular lateral incisors with gingival recession due to trauma from occlusion. (figure i)

Patient's medical history was inconspicuous. There was no familial history of supernumerary tooth or congenitally missing teeth. History of trauma and dental extractions could not be elicited from clinical examination. On radiographic examination there was absence of permanent mandibular central incisor tooth buds (Figure ii).

\section{Case 2}

A10-year-old male patient reported to the department of Pedodontics and preventive dentistry for regular dental check-up. On examination patient's height, weight, built and nourishment corresponds with the chronological age. Intra oral examination of the lower arch, revealed the presence only 3 incisors in the lower arch with no interdental spacing. (Figure iii)

Patient's medical history was inconspicuous. There was no familial history of supernumerary tooth or congenitally missing teeth. History of trauma and dental extractions could not be elicited from clinical examination. On radiographic examination there was absence of permanent mandibular left incisor tooth buds (Figure iv).

\section{Case 3}

An eight year old female patient reported to the department of Pedodontics and Preventive Dentistry with a chief complaint of pain in the upper left back tooth region. On examination patient's height, weight, built and nourishment corresponds with the chronological age. On intraoral examination deep caries with respect to 64 and spacing in the mandibular arch was noticed. Radiographic examination revealed congenital absence of lower central incisors. (Figure v).

\section{Results and Discussion}

Agenesis or missing teeth can be considered when teeth can neither be observed clinically, or radiographically, with any history of trauma or extraction. Occurrence is rare in primary dentition when compared to permanent dentition. Hypodontia as a common feature in many systemic conditions such as Down syndrome, Hypohidrotic ectodermal dysplasia, Chondro-ectodermal dysplasia and Van Der Woude syndrome. ${ }^{9,10}$ 
Int.J.Curr.Res.Aca.Rev.2016; 4(12): 159-163

Fig.1 Absence of lower incisors in the mandibular arch with local gingival inflammation and recession

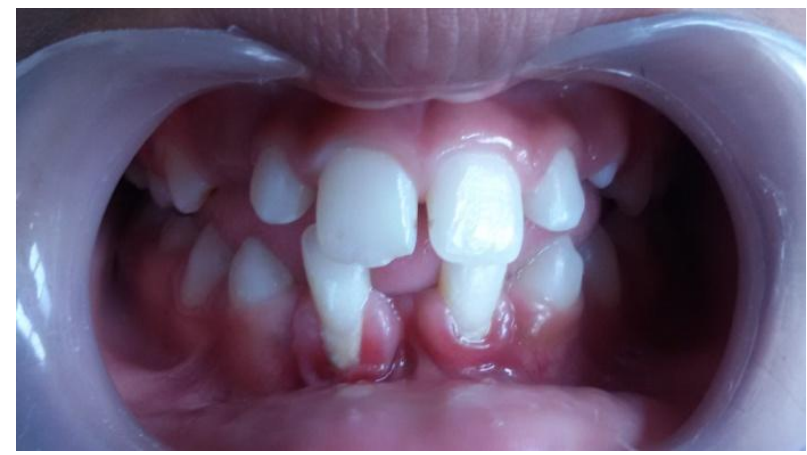

Fig.2 Radiograph showing mandibular central incisor agenesis.

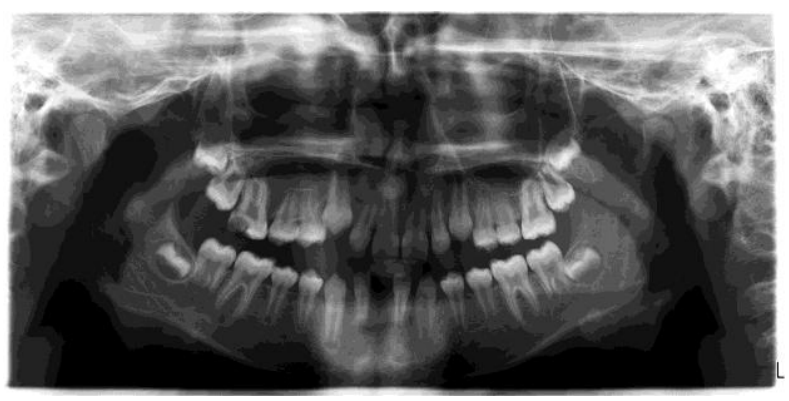

Fig.3 Intraoral view showing presence of only three mandibular incisors.

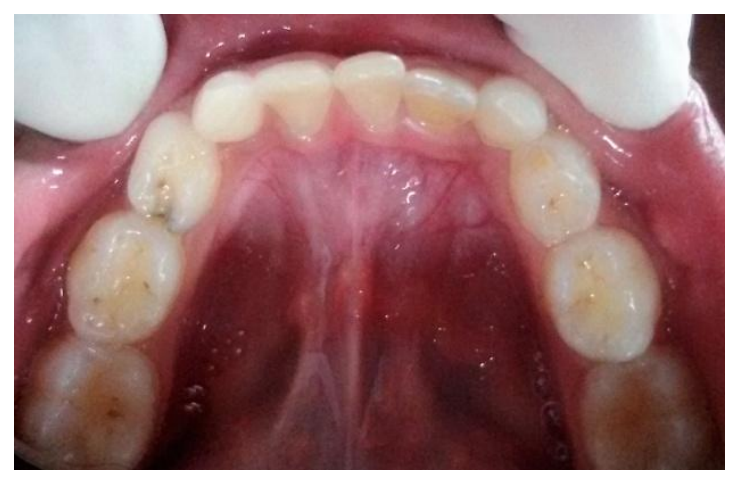

Fig.4 Radiograph showing presence of only three incisors in the mandibular arch.

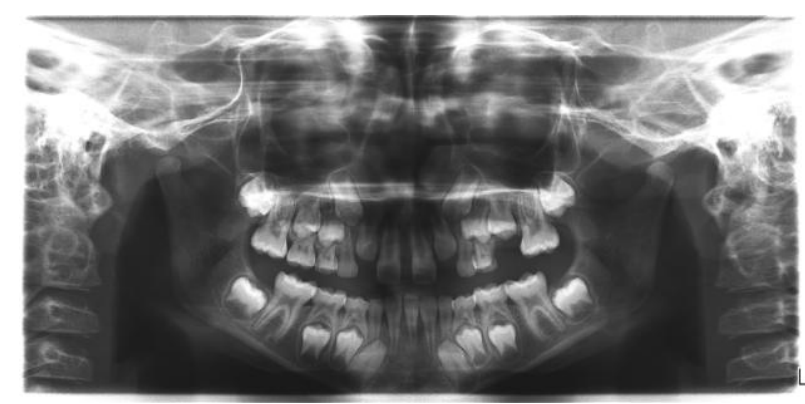


Fig.5 Radiograph showing congenital absence of lower central incisors.

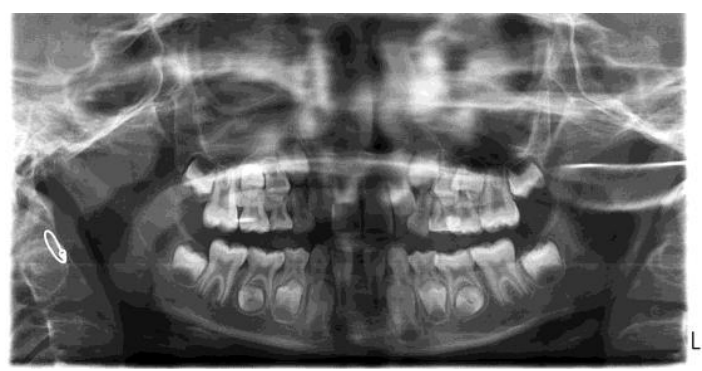

Non-syndromic hypodontia as in our case series calls for a common genetic aetiology. Many researchers have reported that tooth agenesis is associated with palatally displaced maxillary canine, infraocclusion of primary molars, ectopic eruption of molars, delay of tooth development, enamel hypoplasia, and abnormal morphology (pegshaped lateral incisors and reduced dimensions; Lai and Seow, 1989; Garib et al., 2010; Baccetti, 1998; Bjerklin et al., 1992). ${ }^{11}$

Hypodontia along with peg shaped laterals, supernumerary teeth, ectopic eruption of maxillary permanent first molar all considered as micro symptoms of hereditary disturbance which occur due to disturbance in the development of tooth structure. ${ }^{9}$

Several factors like infection, trauma, metabolic disorders, radiation, environmental, genetic factors and idiopathic are considered as possible etiological factors of congenital agenesis. Various theories have been put forth to locate the exact cause of congenital agenesis of incisors. In 1998 Newman and Newman have proposed four theories which mainly explain the cause of agenesis. Firstly, may be due to familial or hereditary distribution. Secondly, during the formation of mandibular symphysis the tooth buds that form the lower incisors could be disturbed leading to agenesis. ${ }^{5}$ Thirdly, failure in attempting to locate itself in the short dental arches and thus leading to reduction in dentition and finally, localized infections or inflammations in the jaws leading to disturbed dental tissue buds causing agenesis. ${ }^{6,10}$ Genetic factors like MSX1 and PAX9 have been explained in the development of incisors. Disturbances in the interaction between these factors are also considered as a main etiologic factor for congenital missing teeth. ${ }^{12}$ Biologically, congenital absence of permanent teeth is attributed to failure in proliferation of lingual or distal tooth buds from the dental lamina. $^{12}$

Accurate diagnosis of a missing tooth requires clinical and radiographic examinations and sometimes dental casts to distinguish whether the tooth is extracted impacted or congenitally absent. ${ }^{13}$

A variety of treatment options exist for congenitally missing teeth. If primary teeth with sufficient root are retained, intentional root canal therapy with gutta-percha followed by crown placement can be done. Other treatment options include auto transplantation, orthodontic space closure, prosthetic rehabilitation with removable partial denture, fixed partial denture or implants.

\section{Conclusion}

Care should be taken to thorough document the clinical history as well as medical and 
Int.J.Curr.Res.Aca.Rev.2016; 4(12): 159-163

radiological examination to rule out any systemic conditions associated with congenitally missing teeth.

\section{References}

1. Mohammad G, Mishra G, Haseebuddin S, Mishra A. 2011. Oligodontia of permanent teeth: A rare case report. Indian J Stomatol;2(4):285-7.

2. Prasanna AL, Manne R, Manne V, Rao S, Ravaneet K. 2012. Congenitally missing permanent teeth: An unusual case. J Oral Res Rev;4(2):56-61.

3. Backman B, Wahlin YB. 2001.Variations in number and morphology of permanent teeth in 7year-old Swedish children. Int $J$ Paediatr Dent.;11:11-17.

4. Endo T, Ozoe R, Kubota M, Akiyama M, Shimooka S. 2006. A survey of hypodontia in Japanese orthodontic patients. Am J Orthod Dentofacial Orthop.;129:29-35.

5. Endo T, Ozoe R, Kojima K, Shimooka S. 2007. Congenitally missing mandibular incisors and mandibular symphysis morphology. The Angle Orthodontist.;77(6):1079-84.

6. Nagaveni NB, Umashankara KV. 2009. Congenital bilateral agenesis of permanent mandibular incisors: case reports and literature review. Arch Orofac Sci;4(2):41-6.
7. Khosravanifard B, GhanbariAzarnir S, Rakhshan H, Sajjadi SH, Ehsan AM, Rakhshan V. 2012. Association between orthodontic treatment need and masticatory performance. Orthodontics (Chic) ;13:e20-8.

8. Rakhshan V. 2015. Congenitally missing teeth (hypodontia): A review of the literature concerning the etiology, prevalence, risk factors, patterns and treatment. Dental research journal.;12(1):1.

9. Thiruvananthapuram, Kerala, and AG1 Guru Royal Palace. 2012. Congenitally missing primary lateral incisors along with permanent successors: a case report. Int J Dent Case Rep;2(1):47-9.

10. Burki S, Rashid S, Bashir U. 2002. Orthodontic management option of congenitally missing mandibular central incisors. Pak Oral Dent J;22(1):19-23.

11. Mirabella AD, Kokich VG, Rosa M. 2011. Analysis of crown widths in subjects with congenitally missing maxillary lateral incisors. The European Journal of Orthodontics. 12:cjr094.

12. Tangade P, Batra M. 2012. Non syndromic oligodontia: Case report. Ethiop J Health Sci;22(3):219-21.

13. Silva Meza R. 2003. Radiographic assessment of congenitally missing teeth in orthodontic patients. Int $\mathrm{J}$ Paediatr Dent.;13:112-6.

\section{How to cite this article:}

Savitha Sathyaprasad, Aiswarya Mohan. 2016. Agenesis of Permanent Mandibular Incisors: Case Series. Int.J.Curr.Res.Aca.Rev.4(12): 159-163. doi: http://dx.doi.org/10.20546/ijcrar.2016.412.015 